\title{
Theoretical foundations of the quantum statistical approach to parton distributions and recent results
}

\author{
Jacques Soffer* \\ Department of Physics, Temple University, Philadelphia, PA 19122-6082, USA \\ E-mail: iacques.sofferdgmail.com
}

\section{Claude Bourrely}

Aix-Marseille Université, Département de Physique, Faculté des Sciences de Luminy,

F-13288 Marseille, Cedex 09, France

E-mail: bourrely@cmi.univ-mrs.fr

\section{Franco Buccella}

INFN, Sezione di Napoli, Via Cintia, Napoli, I-80126, Italy

E-mail: buccelladana.infn.it

The theoretical foundations of the quantum statistical approach to parton distributions are reviewed together with the phenomenological motivations from a few specific features of Deep Inelastic Scattering data. The chiral properties of QCD lead to strong relations between quarks and antiquarks distributions and automatically account for the flavor and helicity symmetry breaking of the sea. We are able to describe both unpolarized and polarized structure functions in terms of a small number of parameters. The extension to include their transverse momentum dependence will be also briefly considered.

XXI International Workshop on Deep-Inelastic Scattering and Related Subject -DIS2013, 22-26 April 2013

Marseilles, France

\footnotetext{
* Speaker.
} 


\section{Basic procedure to construct the statistical parton distributions}

Let us first recall some of the basic ingredients for building up the parton distribution functions (PDF) in the statistical approach, as oppose to the standard polynomial type parametrizations, based on Regge theory at low $x$ and counting rules at large $x$. The fermion distributions are given by the sum of two terms [四], the first one, a quasi Fermi-Dirac function and the second one, a flavor and helicity independent diffractive contribution equal for light quarks. So we have, at the input energy scale $Q_{0}^{2}=4 \mathrm{GeV}^{2}$,

$$
\begin{aligned}
x q^{h}\left(x, Q_{0}^{2}\right) & =\frac{A X_{0 q}^{h} x^{b}}{\exp \left[\left(x-X_{0 q}^{h}\right) / \bar{x}\right]+1}+\frac{\tilde{A} x^{\tilde{b}}}{\exp (x / \bar{x})+1}, \\
x \bar{q}^{h}\left(x, Q_{0}^{2}\right) & =\frac{\bar{A}\left(X_{0 q}^{-h}\right)^{-1} x^{\bar{b}}}{\exp \left[\left(x+X_{0 q}^{-h}\right) / \bar{x}\right]+1}+\frac{\tilde{A} x^{\tilde{b}}}{\exp (x / \bar{x})+1} .
\end{aligned}
$$

It is important to remark that $x$ is indeed the natural variable, since all sum we will use are expressed in terms of $x$. Notice the change of sign of the potentials and helicity for the antiquarks. The parameter $\bar{x}$ plays the role of a universal temperature and $X_{0 q}^{ \pm}$are the two thermodynamical potentials of the quark $q$, with helicity $h= \pm$. It is important to remark that the diffractive contribution occurs only in the unpolarized distributions $q(x)=q_{+}(x)+q_{-}(x)$ and it is absent in the valence $q_{v}(x)=q(x)-\bar{q}(x)$ and in the helicity distributions $\Delta q(x)=q_{+}(x)-q_{-}(x)$ (similarly for antiquarks). The nine free parameters ${ }^{1}$ to describe the light quark sector $\left(u\right.$ and $d$ ), namely $X_{u}^{ \pm}$, $X_{d}^{ \pm}, b, \bar{b}, \tilde{b}, \tilde{A}$ and $\bar{x}$ in the above expressions, were determined at the input scale from the comparison with a selected set of very precise unpolarized and polarized Deep Inelastic Scattering (DIS) data [四]. The additional factors $X_{q}^{ \pm}$and $\left(X_{q}^{ \pm}\right)^{-1}$ come from the transverse momentum dependence (TMD), as explained in Ref. [వ] (See below). For the gluons we consider the black-body inspired expression

$$
x G\left(x, Q_{0}^{2}\right)=\frac{A_{G} x^{b_{G}}}{\exp (x / \bar{x})-1},
$$

a quasi Bose-Einstein function, with $b_{G}$, the only free parameter, since $A_{G}$ is determined by the momentum sum rule.

We also assume that, at the input energy scale, the polarized gluon distribution vanishes, so $x \Delta G\left(x, Q_{0}^{2}\right)=0$. For the strange quark distributions, the simple choice made in Ref. [四] was greatly improved in Ref. [3] . Our procedure allows to construct simultaneously the unpolarized quark distributions and the helicity distributions. This is worth noting because it is a very unique situation. Following our first paper in 2002, new tests against experimental (unpolarized and polarized) data turned out to be very satisfactory, in particular in hadronic collisions, as reported in Refs. [䧃, 回].

\section{Some selected recent results}

For illustration, we will just give one recent result, directly related to the determination of the quark distributions from unpolarized DIS at NLO. We display on Fig. 1 (Left), the resulting

\footnotetext{
${ }^{1} A$ and $\bar{A}$ are fixed by the following normalization conditions $u-\bar{u}=2, d-\bar{d}=1$.
} 
unpolarized statistical PDF versus $x$ at $Q^{2}=10 \mathrm{GeV}^{2}$, where $x u_{v}$ is the $u$-quark valence, $x d_{v}$ the $d$ quark valence, with their characteristic maximum around $x=0.3$, and the gluon $x G$. Note that $x G$ is downscaled by a factor 0.05 . They can be compared with the parton distributions as determined by the HERAPDF1.5 QCD NLO fit, shown also in Fig. 1 (Right), and there is a good agreement, except for the gluon which grows faster than the data. The results are from a recent $e p$ collider data from HERA, combined with previously published data and the accuracy is typically in the range of $1.3-2 \%$.
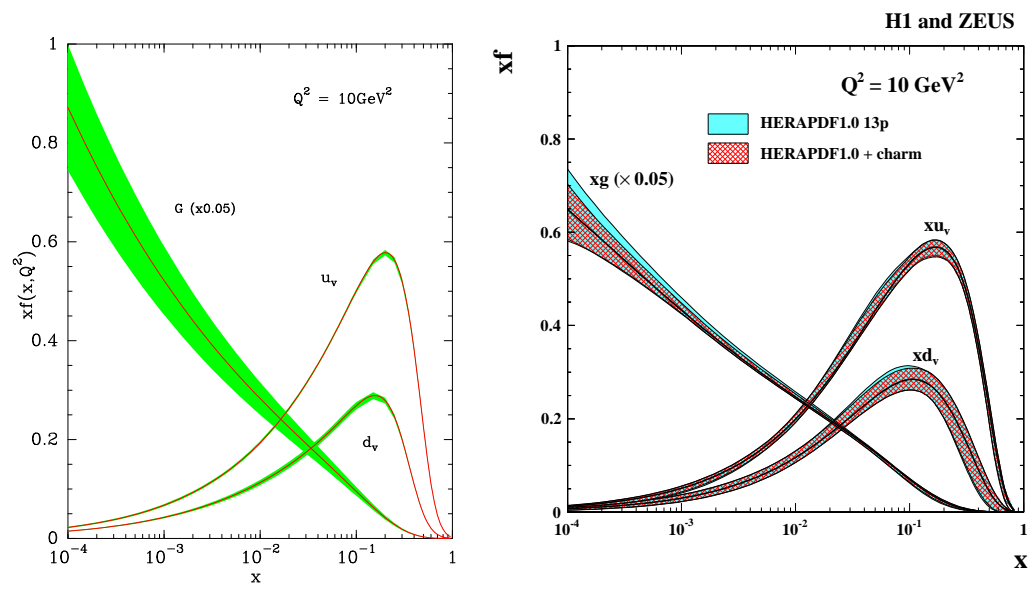

Figure 1: Left: BBS predictions for various statistical unpolarized parton distributions versus $x$ at $Q^{2}=$ $10 \mathrm{GeV}^{2}$. Right : Parton distributions at $Q^{2}=10 \mathrm{GeV}^{2}$, as determined by the HERAPDF fit, with different uncertainties (Taken from Ref. [目]).
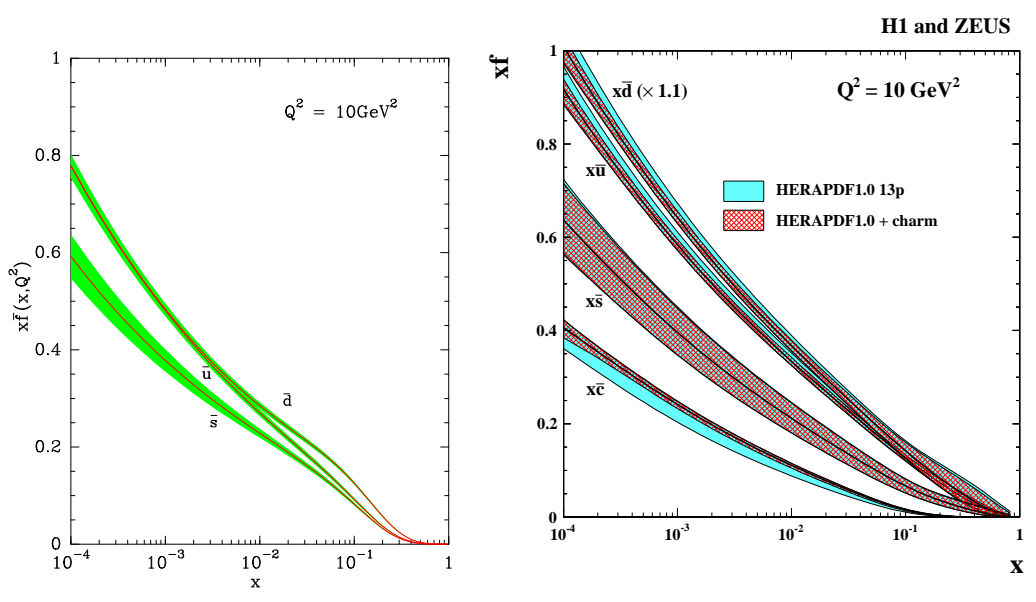

Figure 2: Left : BBS predictions for various statistical unpolarized sea distributions versus $x$ at $Q^{2}=$ $10 \mathrm{GeV}^{2}$. Right : Sea distributions at $Q^{2}=10 \mathrm{GeV}^{2}$, as determined by the HERAPDF fit, with different uncertainties (Taken from Ref. [目]).

We also give a global view of the unpolarized sea distributions displayed in Fig. 2 and we notice that in the small $x$ region $\bar{u}$ and $\bar{d}$ increase less rapidely than the data, whereas $\bar{s}$ agrees 
rather well. Let us comment more on the important question of the flavor asymmetry of the light antiquarks. Our determination of $\bar{u}\left(x, Q^{2}\right)$ and $\bar{d}\left(x, Q^{2}\right)$ is perfectly consistent with the violation of the Gottfried sum rule, for which we found the value $I_{G}=0.2493$ for $Q^{2}=4 \mathrm{GeV}^{2}$. Nevertheless there remains an open problem with the $x$ distribution of the ratio $\bar{d} / \bar{u}$ for $x \geq 0.2$. According to the Pauli principle, this ratio is expected to stay above 1 for any value of $x$. The E866/NuSea Collaboration [ []] has released the final results corresponding to the analysis of their full data set of Drell-Yan yields from an $800 \mathrm{GeV} / \mathrm{c}$ proton beam on hydrogen and deuterium targets and, for $Q^{2}=54 \mathrm{GeV}^{2}$. They obtain the ratio $\bar{d} / \bar{u}$ and, although the errors are rather large in the high- $x$ region, the statistical approach disagrees with a drop off of this ratio for $x>0.2$, suggested by the trend of the data. There is no freedom in the statistical approach, since quark and antiquark distributions are strongly related. On the experimental side, there are now new opportunities for extending the $\bar{d} / \bar{u}$ measurement to larger $x$ up to $x=0.7$, with the running E906 experiment at the $120 \mathrm{GeV}$ Main Injector at Fermilab [ []] and a proposed experiment at the new $30-50 \mathrm{GeV}$ proton accelerator at J-PARC [Q]

For lack of space we cannot show our good results on quark helicity distributions (see Ref. [[5]).

\section{Transverse momentum dependence of the parton distributions}

The parton distributions $p_{i}\left(x, k_{T}^{2}\right)$ of momentum $k_{T}$, must obey the momentum sum rule $\sum_{i} \int_{0}^{1} d x \int x p_{i}\left(x, k_{T}^{2}\right) d k_{T}^{2}=1$, and also the transverse energy sum rule $\sum_{i} \int_{0}^{1} d x \int p_{i}\left(x, k_{T}^{2}\right) \frac{k_{T}^{2}}{x} d k_{T}^{2}=$ $M^{2}$. From the general method of statistical thermodynamics we are led to put $p_{i}\left(x, k_{T}^{2}\right)$ in correspondance with the following expression $\exp \left(\frac{-x}{\bar{x}}+\frac{-k_{T}^{2}}{x \mu^{2}}\right)$, where $\mu^{2}$ is a parameter interpreted as the transverse temperature. So we have now the main ingredients for the extension to the TMD of the statistical PDF. We obtain in a natural way the Gaussian shape with NO $x, k_{T}$ factorization, because the quantum statistics distributions for quarks and antiquarks read in this case

$$
\begin{aligned}
x q^{h}\left(x, k_{T}^{2}\right) & =\frac{F(x)}{\exp \left(x-X_{0 q}^{h}\right) / \bar{x}+1} \frac{1}{\exp \left(k_{T}^{2} / x \mu^{2}-Y_{0 q}^{h}\right)+1}, \\
x \bar{q}^{h}\left(x, k_{T}^{2}\right) & =\frac{\bar{F}(x)}{\exp \left(x+X_{0 q}^{-h}\right) / \bar{x}+1} \frac{1}{\exp \left(k_{T}^{2} / x \mu^{2}+Y_{0 q}^{-h}\right)+1} .
\end{aligned}
$$

Here $F(x)=\frac{A x^{b-1} X_{0 q}^{h}}{\ln \left(1+\exp Y_{0 q}^{h}\right) \mu^{2}}=\frac{A x^{b-1}}{k \mu^{2}}$, where $Y_{0 q}^{h}$ are the thermodynamical potentials chosen such that $\ln \left(1+\exp Y_{0 q}^{h}\right)=k X_{0 q}^{h}$, in order to recover the factors $X_{0 q}^{h}$ and $\left(X_{0 q}^{h}\right)^{-1}$, introduced earlier.

Similarly for $\bar{q}$ we have $\bar{F}(x)=\bar{A} x^{2 b-1} / k \mu^{2}$. The determination of the 4 potentials $Y_{0 q}^{h}$ can be achieved with the choice $k=3.05$. Finally $\mu^{2}$ will be obtained from the transverse energy sum rule and one finds $\mu^{2}=0.198 \mathrm{GeV}^{2}$. Detailed results are shown in Ref. [D]].

Before closing we would like to mention an important point. So far in all our quark or antiquark TMD distributions, the label " ' $h$ "' stands for the helicity along the longitudinal momentum and not along the direction of the momentum, as normally defined for a genuine helicity. The basic effect of a transverse momentum $k_{T} \neq 0$ is the Melosh-Wigner rotation, which mixes the components $q^{ \pm}$in the following way $q^{+M W}=\cos ^{2} \theta q^{+}+\sin ^{2} \theta q^{-}$and $q^{-M W}=\cos ^{2} \theta q^{-}+\sin ^{2} \theta q^{+}$, where, for massless partons, $\theta=\arctan \left(\frac{k_{T}}{p_{0}+p_{z}}\right)$, with $p_{0}=\sqrt{k_{T}^{2}+p_{z}^{2}}$. It vanishes when either $k_{T}=0$ or $p_{z}$, the quark 

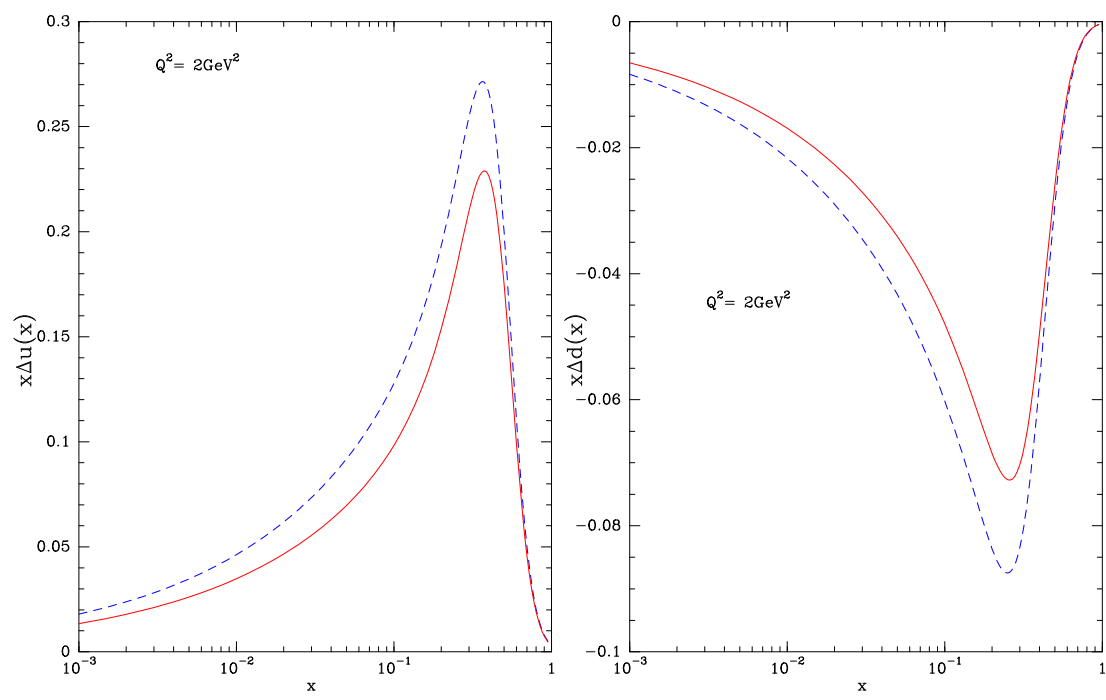

Figure 3: The $u$ and $d$ quark helicity distributions versus $x: x \Delta q(x)$ (dashed line) and $x \Delta q^{M W}(x)$ (solid line). Taken from Ref. [ए0]

longitudinal momentum, goes to infinity. Consequently $q=q^{+}+q^{-}$remains unchanged since $q^{M W}=q$, whereas we have $\Delta q^{M W}=\left(\cos ^{2} \theta-\sin ^{2} \theta\right) \Delta q$.

For illustration we display in Fig. 3, $x \Delta q(x)$ and $x \Delta q^{M W}(x)$ for $Q^{2}=2 \mathrm{GeV}^{2}$, which shows the effect of the Melosh-Wigner rotation, mainly in the low $x$ region.

A new set of PDF is constructed in the framework of a statistical approach of the nucleon. All unpolarized and polarized distributions depend upon a small number of free parameters, with some physical meaning. New tests against experimental (unpolarized and polarized) data on DIS, Semi-inclusive DIS and hadronic processes are very satisfactory. It has a good predictive power but some special features remain to be verified, specially in the high $x$ region. The extension to TMD has been achieved and must be checked more accurately together with Melosh-Wigner effects in the low $x$ region, for small $Q^{2}$.

\section{References}

[1] C. Bourrely, F. Buccella and J. Soffer, Eur. Phys. J. C23, 487 (2002).

[2] C. Bourrely, F. Buccella and J. Soffer, Phys. Rev. D83, 074008 (2011).

[3] C. Bourrely, F. Buccella and J. Soffer, Phys. Lett. B648, 39 (2007).

[4] C. Bourrely, F. Buccella and J. Soffer, Mod. Phys. Lett. A18, 771 (2003).

[5] C. Bourrely, F. Buccella and J. Soffer, Eur. Phys. J. C41, 327 (2005).

[6] F.D. Aaron et al., JHEP 1001, 109 (2010).

[7] R.S. Towell et al., [FNAL E866/Nusea Collaboration], Phys. Rev. D64, 052002 (2001).

[8] D.F. Geesaman et al. [E906 Collaboration], FNAL Proposal E906, April 1, 2001.

[9] J.C.. Peng et al., hep-ph/0007341.

[10] C. Bourrely, F. Buccella and J. Soffer, Journal of Mod. Phys. A28, 1350026 (2013). 